\title{
Rayleigh Confidence Regions based on Record Data
}

\author{
Mousa Abdi ${ }^{\dagger, *}$ and Akbar Asgharzadeh ${ }^{\ddagger}$ \\ $\dagger$ Higher Education Complex of Bam \\ $\ddagger$ University of Mazandaran \\ Received: 8/8/2016 Approved: 10/16/2017
}

\begin{abstract}
This paper presents exact joint confidence regions for the parameters of the Rayleigh distribution based on record data. By providing some appropriate pivotal quantities, we construct several joint confidence regions for the Rayleigh parameters. These joint confidence regions are useful for constructing confidence regions for functions of the unknown parameters. Applications of the joint confidence regions using two environmental data sets are presented for illustrative purposes. Finally, a simulation study is conducted to study the performance of the proposed joint confidence regions.
\end{abstract}

Keywords. Joint confidence region; pivotal quantity; Rayleigh distribution; records.

MSC 2010: 62F25, 62E15.

\section{Introduction}

The Rayleigh distribution was proposed by Rayleigh (1880) in connection with a problem in the field of acoustics. A random variable $X$ is said to have the Rayleigh distribution, if its probability density function (pdf) is given by

$$
f(x ; \mu, \lambda)=2 \lambda(x-\mu) e^{-\lambda(x-\mu)^{2}}, \quad x>\mu, \quad \mu \in R, \quad \lambda>0,
$$

\footnotetext{
* Corresponding author
}

Copyright $(0$ 2019, ASP Ins. This open-access article is published under the terms of the Creative Commons AttributionNonCommercial 4.0 International License which permits Share (copy and redistribute the material in any medium or format) and Adapt (remix, transform, and build upon the material) under the Attribution-NonCommercial terms. 
where $\mu$ and $\lambda$ are the location and scale parameters, respectively. The Rayleigh cumulative distribution function (cdf) is

$$
F(x ; \mu, \lambda)=1-e^{-\lambda(x-\mu)^{2}}, \quad x>\mu, \quad \mu \in R, \quad \lambda>0 .
$$

An important characteristic of the Rayleigh distribution is that its hazard function is an increasing function of time. Therefore, this distribution can be used as a lifetime model in reliability and life testing studies. On the other hand, since the Rayleigh distribution has linearly increasing failure rate, it is an appropriate distribution for a study of components which may not have any manufacturing defects but age rapidly with time. The origin and other applications of this distribution are outlined by Johnson et al. (1994). Some recent references on the Rayleigh distribution are Raqab and Madi (2002), Wu et al. (2006), Soliman and Al-Aboud (2008), Khan et al. (2010), Dey et al. (2014) and Asgharzadeh and Azizpour (2016).

In many real life applications, only observations that exceed or only those that fall below the current extreme value are recorded and the complete data are not available. For example, an electronic component ceases to function in an environment of too high temperature, a battery dies under the stress of time and a wooden beam breaks when sufficient perpendicular force is applied to it (see Ahmadi and Arghami, 2003). Record data are commonly observed in many real life applications involving data relating to weather, sports, economics, environmental studies and life-tests. The statistical study of record values was started with Chandler (1952). Since then, extensive work has done on statistical inference for record data. For more details, see Nevzorov (1988), Ahsanullah (1995) and Arnold et al. (1998). For some recent references, see Asgharzadeh et al. (2013), Mirfarah and Ahmadi (2014) and Zakerzadeh et al. (2016).

Let $\left\{X_{n}, n \geqslant 1\right\}$ be a sequence of independent and identically distributed (iid) random variables with cdf $F(x)$ and pdf $f(x)$. We say an observation $X_{j}$ is an upper record value of this sequence if its value exceeds all previous observations. Thus, $X_{j}$ is an upper record if $X_{j}>X_{i}$ for all $i<j$. If $\left\{T_{n}, n \geqslant 1\right\}$ is defined as: $T_{1}=1$ and $T_{n}=\min \left\{j: j>T_{n-1}, X_{j}>X_{T_{n-1}}\right\}$ for $n \geqslant 2$, then the sequence $\left\{U_{n}=X_{T_{n}}, n \geqslant 1\right\}$ provides a sequence of upper record values from the original sequence $\left\{X_{n}, n \geqslant 1\right\}$. The sequence $\left\{T_{n}, n \geqslant 1\right\}$ represents the record times.

The construction of joint confidence regions for unknown parameters is important in statistical inference. Joint confidence regions are useful for 
constructing confidence bounds for any function of the unknown parameters as well as for testing hypotheses related to unknown parameters. Recently, some authors have discussed the construction of joint confidence regions of unknown parameters based on record data. See, for example, Asgharzadeh and Abdi (2011), Asgharzadeh et al. (2015), Asgharzadeh et al. (2016) and Kinanci et al. (2017). This paper considers joint confidence regions for two-parameter Rayleigh distribution based on record data. By providing some appropriate pivotal quantities, several joint confidence regions for the Rayleigh parameters are first constructed. Optimal joint confidence regions are then proposed.

The rest of the paper is organized as follows. In Section 2, we provide several joint confidence regions for the parameters $\mu$ and $\lambda$. In Section 3, we present illustrative examples using two environmental data sets. A Monte Carlo simulation study is given in Section 4 to compare different proposed joint confidence regions.

\section{Joint Confidence Regions}

In order to construct the joint confidence regions for the parameters $\mu$ and $\lambda$, we use the following lemmas. The proofs are easy and can be found in Arnold et al. (1998).

Lemma 1. Let $U_{1}<U_{2}<\cdots<U_{m}$ be the first $m$ upper record values from a population with $c d f F(\cdot)$. Define

$$
Y_{i}=-\ln \left[1-F\left(U_{i}\right)\right], \quad i=1,2, \ldots, m .
$$

Then $Y_{1}<Y_{2}<\cdots<Y_{m}$ are the first $m$ upper record values from a standard exponential distribution.

Lemma 2. If $Y_{1}<Y_{2}<\cdots<Y_{m}$ are the first $m$ upper record values from the standard exponential distribution. Then, the spacings $Y_{1}, Y_{2}-Y_{1}, \ldots, Y_{m}-$ $Y_{m-1}$ are iid standard exponential variables.

Let $U_{1}<U_{2}<\cdots<U_{m}$ be the first $m$ upper record values from the Rayleigh distribution. Here by providing some appropriate pivotal quantities, we construct several confidence regions for the Rayleigh parameters $\mu$ and $\lambda$. Let us define

$$
Y_{i}=-\ln \left[1-F\left(U_{i}\right)\right]=\lambda\left(U_{i}-\mu\right)^{2} ; \quad i=1,2, \ldots, m .
$$


Then, we have from Lemma 1, that $Y_{1}<Y_{2}<\cdots<Y_{m}$ are the first $m$ upper record values from a standard exponential distribution. Moreover, by Lemma 2 , the spacings $Z_{1}=Y_{1}, Z_{2}=Y_{2}-Y_{1}, \ldots, Z_{m}=Y_{m}-Y_{m-1}$ are iid random variables from the standard exponential distribution. Therefore, the random variable $V_{j}=2 \sum_{i=1}^{j} Z_{i}=2 Y_{j}$ follows a chi-square distribution with $2 j$ degrees of freedom, i.e., $V_{j} \sim \chi_{2 j}^{2}$, and $H_{j}=2 \sum_{i=j+1}^{m} Z_{i}=2\left(Y_{m}-Y_{j}\right) \sim$ $\chi_{2(m-j)}^{2}$, and also that $V_{j}$ and $H_{j}$ are independent, for any $j=1, \ldots, m-1$. In order to derive the confidence regions for the parameters $\mu$ and $\lambda$, we consider the pivotal quantity $T_{j} \equiv T_{j}(\mu)$ defined by

$$
T_{j}(\mu)=\frac{H_{j} / 2(m-j)}{V_{j} / 2 j}=\frac{j}{m-j}\left(\frac{Y_{m}-Y_{j}}{Y_{j}}\right)=\frac{j}{m-j}\left[\frac{\left(U_{m}-\mu\right)^{2}}{\left(U_{j}-\mu\right)^{2}}-1\right],
$$

for $\mu<U_{1}$ and the pivotal quantity

$$
S \equiv S(\mu, \lambda)=H_{j}+V_{j}=2 Y_{m}=2 \lambda\left(U_{m}-\mu\right)^{2} .
$$

Clearly, $T_{j}$ has an $F$ distribution with $2(m-j)$ and $2 j$ degrees of freedom for each $j=1, \ldots, m-1$ and $S \sim \chi_{2 m}^{2}$. Further, by Johnson et al. (1994) (see page 350), $T_{j}$ and $S$ are independent for each $j=1, \ldots, m-1$.

Let for $0<\alpha<1$ that $\chi_{(\alpha),(v)}^{2}$ and $F_{\alpha}\left(v_{1}, v_{2}\right)$ be the upper $\alpha$-quantiles of the $\chi_{(v)}^{2}$ and $\left.F_{(} v_{1}, v_{2}\right)$ distributions, respectively. Next theorem provides $m-1$ exact joint confidence regions for $(\mu, \lambda)$ based on the $m-1$ pairs of pivotal quantities $\left(T_{1}, S\right), \ldots,\left(T_{m-1}, S\right)$.

Theorem 1. Let $U_{1}<U_{2}<\cdots<U_{m}$ be the first $m$ upper record values from the Rayleigh distribution in (1). Then, for any $j=1, \ldots, m-1$, the $100(1-\alpha) \%$ joint confidence region for $(\mu, \lambda)$ is given by

$$
\left\{\begin{aligned}
\frac{U_{j} \sqrt{1+\frac{m-j}{j} F_{\left(\frac{1+\sqrt{1-\alpha}}{2}\right),(2(m-j), 2 j)}}-U_{m}}{\sqrt{1+\frac{m-j}{j} F_{\left(\frac{1+\sqrt{1-\alpha}}{2}\right),(2(m-j), 2 j)}}-1}<\mu<\frac{U_{j} \sqrt{1+\frac{m-j}{j} F_{\left(\frac{1-\sqrt{1-\alpha}}{2}\right),(2(m-j), 2 j)}}-U_{m}}{\sqrt{1+\frac{m-j}{j} F_{\left(\frac{1-\sqrt{1-\alpha}}{2}\right),(2(m-j), 2 j)}}-1}, \\
\frac{\chi_{\left(\frac{1+\sqrt{1-\alpha}}{2}\right),(2 m)}^{2}}{2\left(U_{m}-\mu\right)^{2}}<\lambda<\frac{\chi_{\left(\frac{1-\sqrt{1-\alpha}}{2}\right),(2 m)}^{2}}{2\left(U_{m}-\mu\right)^{2}} .
\end{aligned}\right.
$$

Proof. For any $j=1, \ldots, m-1$, we have

$$
P\left[F_{\left(\frac{1+\sqrt{1-\alpha}}{2}\right),(2(m-j), 2 j)}<T_{j}(\mu)<F_{\left(\frac{1-\sqrt{1-\alpha}}{2}\right),(2(m-j), 2 j)}\right]=\sqrt{1-\alpha}
$$


and

$$
P\left[\chi_{\left(\frac{1+\sqrt{1-\alpha}}{2}\right),(2 m)}^{2}<S(\mu, \lambda)<\chi_{\left(\frac{1-\sqrt{1-\alpha}}{2}\right),(2 m)}^{2}\right]=\sqrt{1-\alpha}
$$

because $T_{j} \sim F_{2(m-j), 2 j}$ and $S \sim \chi_{2 m}^{2}$. Since, for any $j=1, \ldots, m-1, T_{j}$ and $S$ are independent, we conclude that

$$
\begin{aligned}
P\left[F_{\left(\frac{1+\sqrt{1-\alpha}}{2}\right),(2(m-j), 2 j)}<T_{j}<F_{\left(\frac{1-\sqrt{1-\alpha}}{2}\right),(2(m-j), 2 j)},\right. & \\
& \left.\chi_{\left(\frac{1+\sqrt{1-\alpha}}{2}\right),(2 m)}^{2}<S<\chi_{\left(\frac{1-\sqrt{1-\alpha}}{2}\right),(2 m)}^{2}\right]=1-\alpha .
\end{aligned}
$$

By solving the inequalities for $\mu$ and $\sigma$, the $m-1$ joint confidence regions for $(\mu, \lambda)$ can be obtained easily as given in Theorem 1 .

Remark 1. Based on the pivot $T_{j}(j=1, \ldots, m-1)$, a $100(1-\alpha) \%$ confidence interval for $\mu$ is given by

$$
\left(\frac{U_{j} \sqrt{1+\frac{m-j}{j} F_{\left(1-\frac{\alpha}{2}\right),(2(m-j), 2 j)}}-U_{m}}{\sqrt{1+\frac{m-j}{j} F_{\left(1-\frac{\alpha}{2}\right),(2(m-j), 2 j)}}-1}, \frac{U_{j} \sqrt{1+\frac{m-j}{j} F_{\left(\frac{\alpha}{2}\right),(2(m-j), 2 j)}}-U_{m}}{\sqrt{1+\frac{m-j}{j} F_{\left(\frac{\alpha}{2}\right),(2(m-j), 2 j)}}-1}\right) .
$$

Based on the $m-1$ joint confidence regions mentioned in Theorem 1, one can select the optimal confidence region in term of the minimum confidence area. The obtained confidence regions depend only to the extreme records $U_{j}$ and $U_{m}$. But it is reasonable that all of the sample information should be used to obtain confidence regions. For this reason, we consider here another pivotal quantity. Define

$$
Q=\sum_{j=1}^{m-1} T_{j}(\mu)=\sum_{j=1}^{m-1} \frac{j}{m-j}\left[\frac{\left(U_{m}-\mu\right)^{2}}{\left(U_{j}-\mu\right)^{2}}-1\right], \quad \mu<U_{1} .
$$

It is easy to show that the distribution of $Q$ does not depend on $(\mu, \lambda)$. Further, $Q$ and $S$ are independent. Now, using the joint pivot $(Q, S)$, an exact joint confidence region for $(\mu, \lambda)$ can be constructed. To this, we need the following lemma.

Lemma 3. Suppose that $\mu<c_{1}<c_{2}<\cdots<c_{m}$, where $c_{1}, \ldots, c_{m}$ are real 
constants. Let

$$
Q(\mu, m)=\sum_{j=1}^{m-1} \frac{j}{m-j}\left[\frac{\left(c_{m}-\mu\right)^{2}}{\left(c_{j}-\mu\right)^{2}}-1\right]
$$

Then,

(a) $Q(\mu, m)$ is strictly increasing in $\mu$ for any $\mu \in\left(-\infty, c_{1}\right)$.

(b) For $0<t<\infty$, the equation, $Q(\mu, m)=t$ has a unique solution in $\mu$.

Proof. a) The first derivative of $Q(\mu, m)$ with respect to $\mu$ is

$$
\frac{d}{d \mu} Q(\mu, m)=2 \sum_{j=1}^{m-1} \frac{j}{m-j} \cdot \frac{\left(c_{m}-\mu\right)^{2}-\left(c_{m}-\mu\right)\left(c_{j}-\mu\right)}{\left(c_{j}-\mu\right)^{3}} .
$$

Since $\mu<c_{1}<\cdots<c_{m}$, we have $\left(c_{m}-\mu\right)^{2} \geqslant\left(c_{m}-\mu\right)\left(c_{j}-\mu\right)$ for any $j=1,2, \ldots, m-1$, and hence $\frac{d}{d \mu} Q(\mu, m) \geqslant 0$. This complete the proof.

b) Since the function $Q(\mu, m)$ is positive and increasing in $\mu$ with

$\lim _{\mu \rightarrow-\infty} Q(\mu, m)=0 \quad$ and $\quad \lim _{\mu \rightarrow c_{1}} Q(\mu, m)=\sum_{j=1}^{m-1} \frac{j}{m-j}\left[\frac{\left(c_{m}-c_{1}\right)^{2}}{\left(c_{j}-c_{1}\right)^{2}}-1\right]=\infty$,

then $Q(\mu, m)=t$ has a unique solution in $\mu$, for any $0<t<\infty$.

Now, let $q_{\alpha(m)}$ be the upper $\alpha$-quantile of the distribution of the pivotal quantity $Q$. The following theorem provides an exact joint confidence region for $(\mu, \lambda)$ based on the pivot $(Q, S)$.

Theorem 2. Let $U_{1}<U_{2}<\cdots<U_{m}$ be the first $m$ upper record values from the Rayleigh distribution. Then, the following inequalities determine a $100(1-\alpha) \%$ joint confidence region for $(\mu, \lambda)$ based on the pivot $(Q, S)$ :

$$
\left\{\begin{array}{c}
\psi\left(U_{1}, \ldots, U_{m}, q_{\frac{1+\sqrt{1-\alpha}}{2}(m)}\right)<\mu<\psi\left(U_{1}, \ldots, U_{m}, q_{\frac{1-\sqrt{1-\alpha}}{2}(m)}\right), \\
\frac{\chi_{\left(\frac{1+\sqrt{1-\alpha}}{2}\right),(2 m)}^{2}}{2\left(U_{m}-\mu\right)^{2}}<\lambda<\frac{\chi_{\left(\frac{1-\sqrt{1-\alpha}}{2}\right),(2 m)}^{2}}{2\left(U_{m}-\mu\right)^{2}}
\end{array}\right.
$$

where $\psi\left(U_{1}, \ldots, U_{m}, t\right)$ is the solution of $\mu$ for the equation 
Table 1. The upper $\alpha$-quantiles $q_{\alpha(m)}$ of $Q(\mu, m)$

\begin{tabular}{|c|c|c|c|c|c|c|c|c|c|c|}
\hline \multirow[b]{2}{*}{$m$} & \\
\hline & 0.99 & 0.01 & 0.9873 & 0.0127 & 0.975 & 0.025 & 0.95 & 0.05 & 0.90 & 0.10 \\
\hline 2 & 0.0095 & 99.6235 & 0.0126 & 83.3915 & 0.0259 & 40.7528 & 0.0524 & 19.5492 & 0.1144 & 9.0890 \\
\hline 3 & 0.1225 & 104.4499 & 0.1373 & 86.5422 & 0.1984 & 48.0786 & 0.3092 & 25.9073 & 0.4997 & 13.6108 \\
\hline 4 & 0.3185 & 110.5864 & 0.3564 & 92.6991 & 0.5033 & 52.5265 & 0.7128 & 28.8446 & 1.0349 & 16.6134 \\
\hline 5 & 0.6623 & 115.3071 & 0.7215 & 96.2692 & 0.9291 & 53.4702 & 1.2095 & 31.7062 & 1.6459 & 19.2498 \\
\hline 6 & 0.9329 & 114.5048 & 1.0154 & 92.3632 & 1.2925 & 54.4834 & 1.6854 & 33.4537 & 2.2518 & 21.4259 \\
\hline 7 & 1.3339 & 138.2313 & 1.4132 & 110.5868 & 1.7975 & 62.2852 & 2.2611 & 37.3436 & 2.9450 & 23.3300 \\
\hline 8 & 1.7990 & 130.7326 & 1.8845 & 103.4271 & 2.2811 & 60.6067 & 2.8465 & 37.3073 & 3.6175 & 24.8576 \\
\hline 9 & 2.2972 & 126.3819 & 2.4371 & 104.6259 & 2.8304 & 64.2165 & 3.4337 & 39.6563 & 4.3004 & 26.7135 \\
\hline 10 & 2.7129 & 118.5268 & 2.8487 & 100.6608 & 3.3410 & 62.4510 & 4.0277 & 41.1799 & 5.0616 & 28.7925 \\
\hline 11 & 3.1652 & 114.5046 & 3.3151 & 99.7138 & 3.9176 & 62.3512 & 4.6887 & 41.3245 & 5.8162 & 29.3685 \\
\hline 12 & 3.5793 & 143.5688 & 3.7625 & 113.8862 & 4.4965 & 68.4054 & 5.3484 & 44.6854 & 6.5063 & 31.2368 \\
\hline 13 & 4.2893 & 134.5849 & 4.4793 & 114.5675 & 5.0818 & 70.2351 & 5.8937 & 46.5693 & 7.2046 & 33.1427 \\
\hline 14 & 4.7887 & 133.9197 & 5.0214 & 103.4453 & 5.6831 & 66.9488 & 6.6637 & 46.0921 & 7.9878 & 34.0953 \\
\hline 15 & 5.3406 & 137.5944 & 5.6308 & 114.1457 & 6.3912 & 70.8524 & 7.4715 & 48.9024 & 8.8551 & 35.4491 \\
\hline 16 & 5.8045 & 128.6795 & 6.0754 & 111.9826 & 6.9524 & 73.8603 & 8.0119 & 49.8351 & 9.5002 & 36.9925 \\
\hline 17 & 6.5094 & 137.0948 & 6.7653 & 112.5050 & 7.6366 & 71.7216 & 8.7618 & 50.7843 & 10.2887 & 37.8498 \\
\hline 18 & 7.0301 & 124.2017 & 7.3233 & 104.2625 & 8.2129 & 71.4663 & 9.4661 & 51.6745 & 11.1041 & 39.0846 \\
\hline 19 & 7.6386 & 130.0477 & 7.9189 & 108.5718 & 8.9345 & 74.0479 & 10.2628 & 53.0135 & 11.9386 & 40.9066 \\
\hline 20 & 8.1163 & 140.4943 & 8.4362 & 116.1457 & 9.4578 & 77.0187 & 10.7905 & 55.2844 & 12.6170 & 42.5622 \\
\hline 21 & 8.7996 & 142.9720 & 9.1562 & 121.8273 & 10.2679 & 77.3027 & 11.5815 & 56.7319 & 13.5124 & 43.9248 \\
\hline 22 & 9.3456 & 144.5844 & 9.7339 & 121.5262 & 10.9180 & 79.8690 & 12.3567 & 57.7689 & 14.2724 & 44.7949 \\
\hline 23 & 10.1114 & 140.0154 & 10.4640 & 117.9485 & 11.6041 & 79.7559 & 13.0181 & 58.5093 & 15.0332 & 46.1700 \\
\hline 24 & 10.7637 & 146.8277 & 11.1783 & 126.1649 & 12.3699 & 80.9380 & 13.8670 & 60.4229 & 15.9385 & 47.7764 \\
\hline 25 & 11.3202 & 131.0800 & 11.6991 & 112.9332 & 12.9891 & 79.3259 & 14.5160 & 60.7625 & 16.6909 & 48.7578 \\
\hline 30 & 14.8479 & 141.9864 & 15.2383 & 124.6956 & 16.6703 & 86.6005 & 18.3592 & 67.2761 & 20.8426 & 54.4106 \\
\hline 40 & 21.3058 & 168.6216 & 21.8866 & 143.6941 & 23.7145 & 99.7097 & 25.9825 & 79.9148 & 29.0420 & 67.0554 \\
\hline 50 & 28.4495 & 168.4166 & 29.0802 & 148.1588 & 31.2146 & 111.0425 & 33.9035 & 92.6934 & 37.3505 & 79.0237 \\
\hline
\end{tabular}

$$
\sum_{j=1}^{m-1} \frac{j}{m-j}\left[\frac{\left(U_{m}-\mu\right)^{2}}{\left(U_{j}-\mu\right)^{2}}-1\right]=t .
$$

Proof. We have

$$
P\left[q_{\left(\frac{1+\sqrt{1-\alpha}}{2}\right)}<Q<q_{\left(\frac{1-\sqrt{1-\alpha}}{2}\right)}\right]=\sqrt{1-\alpha}
$$

and

$$
P\left[\chi_{\left(\frac{1+\sqrt{1-\alpha}}{2}\right),(2 m)}^{2}<S<\chi_{\left(\frac{1-\sqrt{1-\alpha}}{2}\right),(2 m)}^{2}\right]=\sqrt{1-\alpha} .
$$


By independence of $Q$ and $S$, we obtain

$P\left[q_{\left(\frac{1+\sqrt{1-\alpha}}{2}\right)}<Q<q_{\left(\frac{1-\sqrt{1-\alpha}}{2}\right)}, \chi_{\left(\frac{1+\sqrt{1-\alpha}}{2}\right),(2 m)}^{2}<S<\chi_{\left(\frac{1-\sqrt{1-\alpha}}{2}\right),(2 m)}^{2}\right]=1-\alpha$.

Now by Lemma 3, we can find the joint confidence region as described in Theorem 2.

Remark 2. The $100(1-\alpha) \%$ confidence interval for $\mu$ based on the pivot $Q$ is

$$
\psi\left(U_{1}, \ldots, U_{m}, q_{1-\frac{\alpha}{2}(m)}\right)<\mu<\psi\left(U_{1}, \ldots, U_{m}, q_{\frac{\alpha}{2}(m)}\right)
$$

where $\psi\left(U_{1}, \ldots, U_{m}, t\right)$ is the solution of $\mu$ for the equation

$$
\sum_{j=1}^{m-1} \frac{j}{m-j}\left[\frac{\left(U_{m}-\mu\right)^{2}}{\left(U_{j}-\mu\right)^{2}}-1\right]=t .
$$

It should be mentioned here that since the exact distribution of the pivotal quantity $Q$ is too hard to derive algebraically, we need to compute the upper $\alpha$-quantile $q_{\alpha(m)}$ by using Monte Carlo simulation. In Table 1, we presented the upper $\alpha$-quantile $q_{\alpha(m)}$ of $Q$ for $m=2,3, \ldots, 25,30,40,50$ and various values of $\alpha$ over 20000 replications.

\section{Numerical Examples}

In this section, two examples from wind speed data and annual rainfall data are given to illustrate the proposed joint confidence regions.

\section{Example 1: Wind speed data}

Let us first consider the wind speed data reported by Battacharya and Bhattacharjee (2010). The data are the average monthly wind speed (m/s) of Kolkata from 1st March 2009 to 31st March 2009.

$\begin{array}{llllllllllll}0.56 & 0.28 & 0.56 & 0.56 & 1.11 & 0.83 & 1.11 & 1.94 & 1.11 & 0.83 & 1.11\end{array}$

$\begin{array}{llllllllllll}1.39 & 0.28 & 0.56 & 0.28 & 0.28 & 0.28 & 0.83 & 1.39 & 1.11 & 1.11 & 0.83\end{array}$

$\begin{array}{llllllllll}0.56 & 0.83 & 1.67 & 1.94 & 1.39 & 0.83 & 2.22 & 1.67 & 2.22 .\end{array}$

We have checked the validity of the Rayleigh distribution based on the parameters $\mu=0.0225$ and $\lambda=0.75911$, using the Kolmogorov-Smirnov (K-S) 
Table 2. The areas of $95 \%$ confidence regions for $(\mu, \lambda)$ in Example 1.

\begin{tabular}{lcccc}
\hline \hline Pivot & $\left(T_{1}, S\right)$ & $\left(T_{2}, S\right)$ & $\left(T_{3}, S\right)$ & $(Q, S)$ \\
\hline Region & $A_{1}$ & $A_{2}$ & $A_{3}$ & $A_{4}$ \\
\hline Area & 4.31223 & 5.05170 & 5.25395 & 1.97945 \\
\hline
\end{tabular}

test. It is observed that the K-S distance is $\mathrm{K}-\mathrm{S}=0.1257$ with a corresponding $\mathrm{p}$-value $=0.6655$. This shows that the Rayleigh model fits well to the above data.

If only the upper record values have been observed, these are as follows:

$$
\begin{array}{llll}
0.56 & 1.11 & 1.94 & 2.22 .
\end{array}
$$

Now, we use the methods proposed in Section 2 to construct the exact joint confidence regions for $(\mu, \lambda)$. To obtain the $95 \%$ joint confidence regions, we need the following percentiles:

$$
\begin{aligned}
F_{0.0127(6,2)} & =78.07254, & F_{0.9873(6,2)} & =0.1014364, \\
F_{0.0127(4,4)} & =14.02461, & F_{0.9873(4,4)} & =0.07130322, \\
F_{0.0127(2,6)} & =9.858393, & F_{0.9873(2,6)} & =0.0128086, \\
q_{0.0127(4)} & =92.6991, & q_{0.9873(4)} & =0.3564 \\
\chi_{0.0127(8)}^{2} & =19.4347, & \chi_{0.9873(8)}^{2} & =1.768713 .
\end{aligned}
$$

By Theorem 1 and using the S-PLUS package, the $95 \%$ joint confidence regions for $(\mu, \lambda)$ based on the pivots $\left(T_{1}, S\right),\left(T_{2}, S\right)$ and $\left(T_{3}, S\right)$ are given respectively by

$$
\begin{aligned}
& A_{1}=\left\{(\mu, \lambda):-11.1249<\mu<0.4442, \quad \frac{1.76871}{2(2.22-\mu)^{2}}<\lambda<\frac{19.4347}{2(2.22-\mu)^{2}}\right\}, \\
& A_{2}=\left\{(\mu, \lambda):-30.5701<\mu<0.5600, \quad \frac{1.76871}{2(2.22-\mu)^{2}}<\lambda<\frac{19.4347}{2(2.22-\mu)^{2}}\right\}, \\
& A_{3}=\left\{(\mu, \lambda):-129.3617<\mu<0.5600, \quad \frac{1.76871}{2(2.22-\mu)^{2}}<\lambda<\frac{19.4347}{2(2.22-\mu)^{2}}\right\} .
\end{aligned}
$$



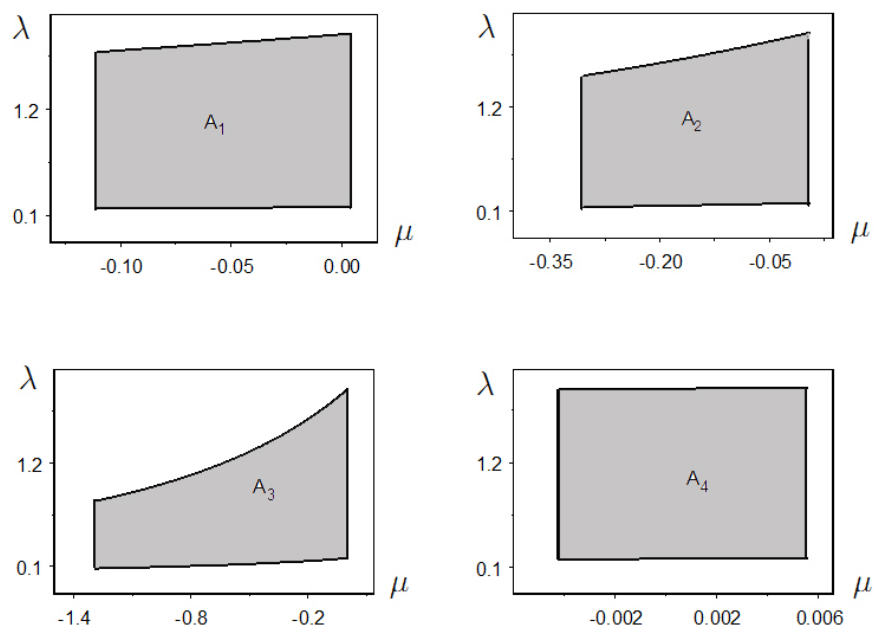

Figure 1. The $95 \%$ joint confidence region for $(\mu, \lambda)$ in Example 1.

By Theorem 2, the $95 \%$ joint confidence region based on the pivot $(Q, S)$ is

$A_{4}=\left\{(\mu, \lambda):-0.4233<\mu<0.5600, \quad \frac{1.76871}{2(2.22-\mu)^{2}}<\lambda<\frac{19.4347}{2(2.22-\mu)^{2}}\right\}$,

In Table 2, we have presented the areas of the $95 \%$ joint confidence regions based on different pivots. From Table 2, it is observed that the region $A_{4}$ has the smallest area and hence, the pivot $(Q, S)$ is the optimal pivotal quantity. Figure 1 shows the shapes of different Rayleigh confidence regions for $\mu$ and $\lambda$.

\section{Example 2: Annual rainfall data}

In this example, we present a data analysis of the amount of annual rainfall (in inches) recorded at the Los Angeles Civic Center for 50 years, from 1962 to 2012 (season July 1-June 30). See the website of Los Angeles Almanac: www.laalmanac.com/weather/we08aa.htm. The validity of the Rayleigh model to these data can be checked using the K-S test. For these data, the observed rainfall records are as follows:

$$
8.38,13.68,20.44,22.00,27.47,33.44,37.96 \text {. }
$$

By Theorem 1, the $95 \%$ joint confidence regions for $(\mu, \lambda)$ based on the pivots 
Table 3. The areas of $95 \%$ joint confidence regions for $(\mu, \lambda)$ in Example 2.

\begin{tabular}{lccccccc}
\hline \hline Pivot & $\left(T_{1}, S\right)$ & $\left(T_{2}, S\right)$ & $\left(T_{3}, S\right)$ & $\left(T_{4}, S\right)$ & $\left(T_{5}, S\right)$ & $\left(T_{6}, S\right)$ & $(Q, S)$ \\
\hline Region & $B_{1}$ & $B_{2}$ & $B_{3}$ & $B_{4}$ & $B_{5}$ & $B_{6}$ & $B_{7}$ \\
\hline Area & 0.267077 & 0.315092 & 0.331864 & 0.362139 & 0.379825 & 0.394150 & 0.129316 \\
\hline
\end{tabular}

$\left(T_{j}, S\right), j=1, \ldots, 6$ are respectively as:

$B_{1}=\left\{(\mu, \lambda):-67.3112<\mu<6.9495, \quad \frac{4.88886}{2(37.96-\mu)^{2}}<\lambda<\frac{28.37037}{2(37.96-\mu)^{2}}\right\}$

$B_{2}=\left\{(\mu, \lambda):-105.529<\mu<8.3800, \quad \frac{4.88886}{2(37.96-\mu)^{2}}<\lambda<\frac{28.37037}{2(37.96-\mu)^{2}}\right\}$,

$B_{3}=\left\{(\mu, \lambda):-142.525<\mu<8.3800, \quad \frac{4.88886}{2(37.96-\mu)^{2}}<\lambda<\frac{28.37037}{2(37.96-\mu)^{2}}\right\}$,

$B_{4}=\left\{(\mu, \lambda):-299.6522<\mu<8.3800, \quad \frac{4.88886}{2(37.96-\mu)^{2}}<\lambda<\frac{28.37037}{2(37.96-\mu)^{2}}\right\}$,

$B_{5}=\left\{(\mu, \lambda):-649.0486<\mu<8.3800, \quad \frac{4.88886}{2(37.96-\mu)^{2}}<\lambda<\frac{28.37037}{2(37.96-\mu)^{2}}\right\}$,

$B_{6}=\left\{(\mu, \lambda):-4207.989<\mu<8.3800, \quad \frac{4.88886}{2(37.96-\mu)^{2}}<\lambda<\frac{28.37037}{2(37.96-\mu)^{2}}\right\}$.

Also by Theorem 2 , the $95 \%$ joint confidence region based on the pivot $(Q, S)$ is

$B_{7}=\left\{(\mu, \lambda):-5.91444<\mu<8.3800, \quad \frac{4.88886}{2(37.96-\mu)^{2}}<\lambda<\frac{28.37037}{2(37.96-\mu)^{2}}\right\}$.

Table 3 shows the areas of different confidence regions for the parameters 

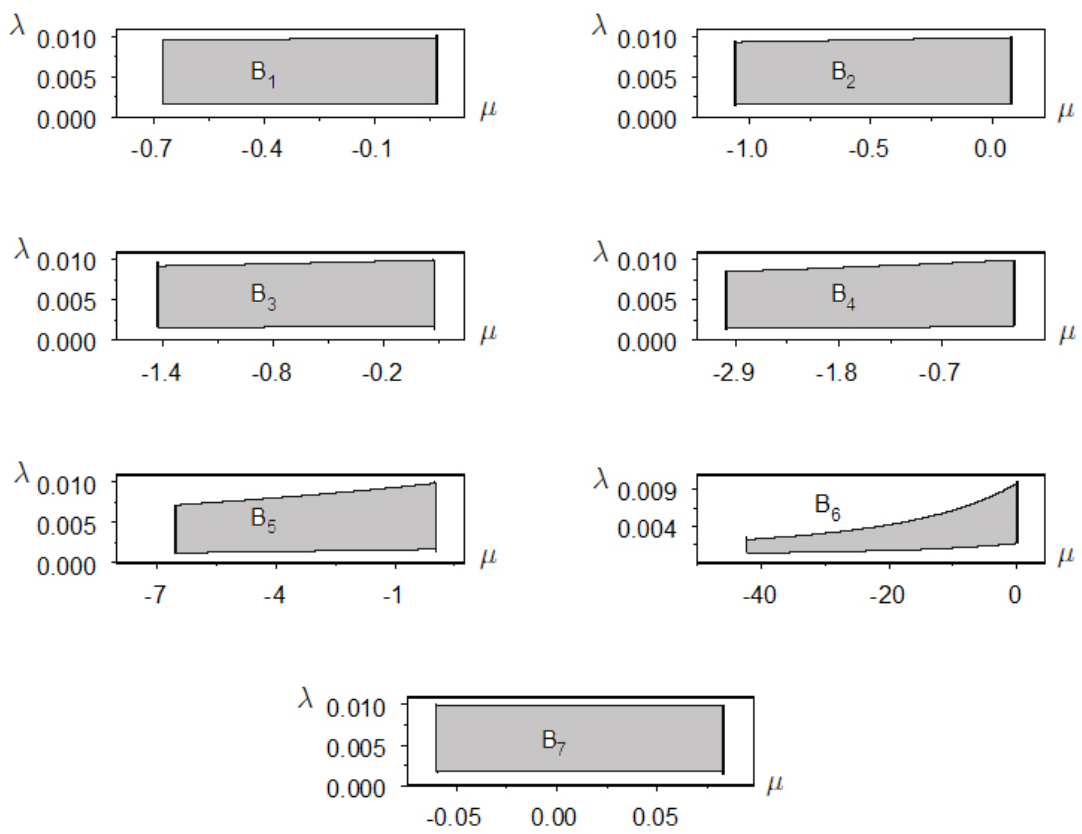

Figure 2. The 95\% joint confidence region for $(\mu, \lambda)$ in Example 2.

$\mu$ and $\lambda$. As we can see from Table 3 , the pivot $(Q, S)$ provides again the smallest confidence area. The shapes of different joint confidence regions are given in Figure 2.

\section{Simulation Study}

In this section, we carry out a Monte Carlo simulation to compare the performances of the different confidence regions. Before progressing further, first we describe how we can generate an upper record sample from any continuous distribution. The upper record sample $U_{1}, U_{2}, \ldots, U_{m}$ from any continuous cdf $F(\cdot)$ can be generated using the following simulational algorithm:

step 1. Generate $m$ independent $\operatorname{Exp}(1)$ observations $X_{1}, \ldots, X_{m}$.

step 2. Set $R_{i}=X_{1}+X_{2}+\cdots+X_{i}$ for $i=1,2, \ldots, m$.

step 3. Set $W_{i}=1-e^{-R_{i}}$ for $i=1,2, \ldots, m$. Then $W_{1}, W_{2}, \ldots, W_{m}$ is the required upper record sample from the Uniform $(0,1)$ distribution. 
Table 4 . The average area and coverage probability of $95 \%$ joint confidence region for $(\mu, \lambda)$.

\begin{tabular}{|c|c|c|c|}
\hline$m$ & Pivots & $\mathrm{CP}$ & $\mathrm{CA}$ \\
\hline \multirow[t]{5}{*}{5} & $\left(T_{1}, S\right)$ & 0.950 & 7.0787 \\
\hline & $\left(T_{2}, S\right)$ & 0.949 & 7.6066 \\
\hline & $\left(T_{3}, S\right)$ & 0.945 & 8.1522 \\
\hline & $\left(T_{4}, S\right)$ & 0.953 & 8.6028 \\
\hline & $(\mathrm{Q}, \mathrm{S})$ & 0.975 & 6.2913 \\
\hline \multirow[t]{10}{*}{10} & $\left(T_{1}, S\right)$ & 0.946 & 3.9620 \\
\hline & $\left(T_{2}, S\right)$ & 0.952 & 4.1724 \\
\hline & $\left(T_{3}, S\right)$ & 0.946 & 4.3949 \\
\hline & $\left(T_{4}, S\right)$ & 0.948 & 4.6089 \\
\hline & $\left(T_{5}, S\right)$ & 0.951 & 4.8923 \\
\hline & $\left(T_{6}, S\right)$ & 0.953 & 5.1965 \\
\hline & $\left(T_{7}, S\right)$ & 0.954 & 5.4924 \\
\hline & $\left(T_{8}, S\right)$ & 0.951 & 5.8604 \\
\hline & $\left(T_{9}, S\right)$ & 0.949 & 5.9651 \\
\hline & $(\mathrm{Q}, \mathrm{S})$ & 0.971 & 3.6330 \\
\hline \multirow[t]{15}{*}{15} & $\left(T_{1}, S\right)$ & 0.948 & 2.9786 \\
\hline & $\left(T_{2}, S\right)$ & 0.952 & 3.1128 \\
\hline & $\left(T_{3}, S\right)$ & 0.953 & 3.2664 \\
\hline & $\left(T_{4}, S\right)$ & 0.951 & 3.4132 \\
\hline & $\left(T_{5}, S\right)$ & 0.950 & 3.5339 \\
\hline & $\left(T_{6}, S\right)$ & 0.948 & 3.6665 \\
\hline & $\left(T_{7}, S\right)$ & 0.948 & 3.8194 \\
\hline & $\left(T_{8}, S\right)$ & 0.948 & 3.9919 \\
\hline & $\left(T_{9}, S\right)$ & 0.949 & 4.1693 \\
\hline & $\left(T_{10}, S\right)$ & 0.951 & 4.3584 \\
\hline & $\left(T_{11}, S\right)$ & 0.946 & 4.6193 \\
\hline & $\left(T_{12}, S\right)$ & 0.949 & 4.9115 \\
\hline & $\left(T_{13}, S\right)$ & 0.948 & 5.2216 \\
\hline & $\left(T_{14}, S\right)$ & 0.950 & 5.4530 \\
\hline & $(\mathbf{Q}, \mathrm{S})$ & 0.970 & 2.9363 \\
\hline
\end{tabular}

\begin{tabular}{cccc}
\hline \hline$m$ & pivots & CP & CA \\
\hline 30 & $\left(T_{1}, S\right)$ & 0.960 & 1.9568 \\
& $\left(T_{2}, S\right)$ & 0.962 & 2.0278 \\
$\left(T_{3}, S\right)$ & 0.954 & 2.0793 \\
$\left(T_{4}, S\right)$ & 0.959 & 2.1376 \\
$\left(T_{5}, S\right)$ & 0.959 & 2.1907 \\
$\left(T_{6}, S\right)$ & 0.958 & 2.2518 \\
$\left(T_{7}, S\right)$ & 0.960 & 2.2989 \\
$\left(T_{8}, S\right)$ & 0.964 & 2.3688 \\
$\left(T_{9}, S\right)$ & 0.959 & 2.4214 \\
$\left(T_{10}, S\right)$ & 0.958 & 2.4657 \\
$\left(T_{11}, S\right)$ & 0.961 & 2.5465 \\
$\left(T_{12}, S\right)$ & 0.961 & 2.5927 \\
$\left(T_{13}, S\right)$ & 0.957 & 2.6392 \\
$\left(T_{14}, S\right)$ & 0.963 & 2.7086 \\
$\left(T_{15}, S\right)$ & 0.960 & 2.7786 \\
$\left(T_{16}, S\right)$ & 0.965 & 2.8671 \\
$\left(T_{17}, S\right)$ & 0.964 & 2.9455 \\
$\left(T_{18}, S\right)$ & 0.962 & 3.0169 \\
$\left(T_{19}, S\right)$ & 0.959 & 3.1041 \\
$\left(T_{20}, S\right)$ & 0.957 & 3.2078 \\
$\left(T_{21}, S\right)$ & 0.965 & 3.3272 \\
$\left(T_{22}, S\right)$ & 0.956 & 3.4182 \\
$\left(T_{23}, S\right)$ & 0.960 & 3.5946 \\
$\left(T_{24}, S\right)$ & 0.962 & 3.7225 \\
$\left(T_{25}, S\right)$ & 0.968 & 3.8965 \\
$\left(T_{26}, S\right)$ & 0.956 & 4.0883 \\
$\left(T_{27}, S\right)$ & 0.961 & 4.3975 \\
$\left(T_{28}, S\right)$ & 0.960 & 4.4012 \\
$\left(T_{29}, S\right)$ & 0.966 & 4.4521 \\
\hline$(\mathbf{Q}, \mathbf{S})$ & $\mathbf{0 . 9 6 9}$ & $\mathbf{1 . 2 4 7 0}$ \\
\hline & & & \\
& & \\
& & \\
&
\end{tabular}


Table 5. Comparison of average confidence area based on complete data and record data.

\begin{tabular}{lcc}
\hline \hline & Complete data & Record data \\
\hline$m=5$ & 3.0069 & 6.2913 \\
$m=10$ & 0.5339 & 3.6330 \\
$m=15$ & 0.5100 & 2.9363 \\
$m=30$ & 0.0244 & 1.2470 \\
\hline
\end{tabular}

step 4. Finally, we set $U_{i}=F^{-1}\left(W_{i}\right)$ for $i=1,2, \ldots, m$. Then $U_{1}, U_{2}, \ldots, U_{m}$ is the required upper record sample from the distribution $F(\cdot)$.

\subsection{Comparison of Confidence Regions}

Using the above algorithm, we have randomly generated 10000 upper record sample $U_{1}, U_{2}, \ldots, U_{m}$ from the standard Rayleigh distribution (i.e., $\mu=0$ and $\lambda=1$ ) and then computed the $95 \%$ confidence regions using Theorems 1 and 2. We then compared the performances of these regions in terms of confidence area (CA) and coverage probability (CP). In Table 4, for different values of $m$, we presented the average confidence areas and coverage probabilities of different joint confidence regions over 10000 replications. All the computations are performed using SPLUS package. The simulation results show that when $m$ increases, the areas are decreased. In addition, the coverage probabilities of the joint confidence regions for $(\mu, \lambda)$ are close to the desired level of 0.95 for different pivots and different record sample sizes. The coverage probabilities based on the joint pivot $(Q, S)$ are slightly larger than the corresponding coverage probabilities based on the other pivots. Comparing the average areas of the confidence regions, it is observed that in all of cases considered, the joint pivot $(Q, S)$ provides the first smallest area for $(\mu, \lambda)$ and the second smallest area for $(\mu, \lambda)$ is provided by the pivot $\left(T_{1}, S\right)$. Based on the simulation results, overall speaking, we would recommend the use of pivot $(Q, S)$ for constructing joint confidence region for the Rayleigh parameters $\mu$ and $\lambda$.

Let us now compare the confidence regions obtained based on first $m$ record data by the confidence regions obtained based on a simple random sample $X_{1}, \ldots, X_{m}$ from the original distribution. In Section 2, the pivot $(Q, S)$ was used for constructing a joint confidence region for $(\mu, \lambda)$ based on the first $m$ upper record data. If $\left(Q^{\prime}, S^{\prime}\right)$ is the corresponding pivot based 
Table 6. The average confidence length (CL) and coverage probability (CP) of the $95 \%$ confidence intervals for $\mu$.

\begin{tabular}{|c|c|c|c|}
\hline $\mathrm{m}$ & Pivots & $\mathrm{CP}$ & $\mathrm{CL}$ \\
\hline \multirow[t]{5}{*}{5} & $T_{1}$ & 0.949 & 4.3941 \\
\hline & $T_{2}$ & 0.950 & 6.9294 \\
\hline & $T_{3}$ & 0.949 & 13.817 \\
\hline & $T_{4}$ & 0.952 & 75.7907 \\
\hline & $\mathrm{Q}$ & 0.922 & 1.4508 \\
\hline \multirow[t]{10}{*}{10} & $T_{1}$ & 0.943 & 2.9520 \\
\hline & $T_{2}$ & 0.947 & 3.5044 \\
\hline & $T_{3}$ & 0.952 & 4.1125 \\
\hline & $T_{4}$ & 0.955 & 4.9648 \\
\hline & $T_{5}$ & 0.957 & 6.0926 \\
\hline & $T_{6}$ & 0.951 & 8.0292 \\
\hline & $T_{7}$ & 0.951 & 11.4532 \\
\hline & $T_{8}$ & 0.947 & 21.5654 \\
\hline & $T_{9}$ & 0.950 & 114.1194 \\
\hline & $\mathrm{Q}$ & 0.924 & 1.5120 \\
\hline \multirow[t]{15}{*}{15} & $T_{1}$ & 0.947 & 2.6119 \\
\hline & $T_{2}$ & 0.953 & 2.9375 \\
\hline & $T_{3}$ & 0.947 & 3.2398 \\
\hline & $T_{4}$ & 0.946 & 3.5408 \\
\hline & $T_{5}$ & 0.951 & 3.8638 \\
\hline & $T_{6}$ & 0.949 & 4.3356 \\
\hline & $T_{7}$ & 0.949 & 4.8865 \\
\hline & $T_{8}$ & 0.952 & 5.6166 \\
\hline & $T_{9}$ & 0.952 & 6.4380 \\
\hline & $T_{10}$ & 0.949 & 7.8484 \\
\hline & $T_{11}$ & 0.946 & 10.2109 \\
\hline & $T_{12}$ & 0.950 & 14.6433 \\
\hline & $T_{13}$ & 0.950 & 26.9286 \\
\hline & $T_{14}$ & 0.952 & 142.3817 \\
\hline & Q & 0.934 & 1.5440 \\
\hline
\end{tabular}

\begin{tabular}{|c|c|c|c|}
\hline $\mathrm{m}$ & pivots & $\mathrm{CP}$ & CL \\
\hline \multirow[t]{30}{*}{30} & $T_{1}$ & 0.951 & 2.2384 \\
\hline & $T_{2}$ & 0.950 & 2.3729 \\
\hline & $T_{3}$ & 0.952 & 2.5097 \\
\hline & $T_{4}$ & 0.953 & 2.626 \\
\hline & $T_{5}$ & 0.950 & 2.7474 \\
\hline & $T_{6}$ & 0.953 & 2.8489 \\
\hline & $T_{7}$ & 0.949 & 2.9707 \\
\hline & $T_{8}$ & 0.951 & 3.0917 \\
\hline & $T_{9}$ & 0.949 & 3.2353 \\
\hline & $T_{10}$ & 0.943 & 3.3629 \\
\hline & $T_{11}$ & 0.949 & 3.5734 \\
\hline & $T_{12}$ & 0.947 & 3.7468 \\
\hline & $T_{13}$ & 0.952 & 3.8811 \\
\hline & $T_{14}$ & 0.952 & 4.1047 \\
\hline & $T_{15}$ & 0.952 & 4.2471 \\
\hline & $T_{16}$ & 0.946 & 4.5696 \\
\hline & $T_{17}$ & 0.955 & 4.8355 \\
\hline & $T_{18}$ & 0.948 & 5.1708 \\
\hline & $T_{19}$ & 0.953 & 5.5137 \\
\hline & $T_{20}$ & 0.954 & 5.9125 \\
\hline & $T_{21}$ & 0.950 & 6.5241 \\
\hline & $T_{22}$ & 0.948 & 7.2477 \\
\hline & $T_{23}$ & 0.948 & 8.0891 \\
\hline & $T_{24}$ & 0.950 & 9.5706 \\
\hline & $T_{25}$ & 0.943 & 11.226 \\
\hline & $T_{26}$ & 0.952 & 14.7232 \\
\hline & $T_{27}$ & 0.942 & 21.0376 \\
\hline & $T_{28}$ & 0.950 & 38.9845 \\
\hline & $T_{29}$ & 0.932 & 206.399 \\
\hline & $\mathbf{Q}$ & 0.935 & 1.5564 \\
\hline
\end{tabular}


on the order statistics $X_{(1)}, \ldots, X_{(m)}$, then using methods similar to those discussed in Section 2, one can also construct a joint confidence region for $(\mu, \lambda)$. In Table 5 , we have compared the confidence areas obtained based on ordered sample (complete) data and record data. As expected, the area obtained based on the complete data is smaller than that the area obtained based on record data.

\subsection{Comparison of Confidence Intervals}

We have also compared the confidence intervals obtained based on the usual F-distributed pivots $T_{j}(\mu)$ (for $j=1,2, \ldots, m-1$ ) and the one that obtained based on the additive pivotal quantity $Q=\sum_{j=1}^{m-1} T_{j}(\mu)$. Table 6 presents the average confidence lengths and coverage probabilities of the $95 \%$ confidence intervals for $\mu$. As we can see from the table, the pivot $Q(\mu, m)$ provides the shortest confidence length. The coverage probabilities of the confidence intervals for $\mu$ are close to the desired level of 0.95 for different pivots and different record sample sizes. Also, the coverage probabilities based on the pivots $T_{j}(\mu)$ are slightly larger than the one that obtained based on the pivot $Q$.

\section{Acknowledgement}

The authors would like to thank the three referees for their valuable comments and suggestions which greatly improved the paper.

\section{References}

Ahmadi, J. and Arghami, N.R. (2003). Comparing the Fisher Information in Record Values and iid Observations. Statistics, 37, 435-441.

Ahsanullah, M. (1995). Introduction to Record Statistics, NOVA Science Publishers Inc., Huntington, New York.

Arnold, B. C., Balakrishnan, N. and Nagaraja, H. N. (1998), Records. John Wiley \& Sons, New York.

Asgharzadeh, A. and Abdi, M. (2011). Confidence Intervals and Joint Confidence Regions for the Two-parameter Exponential Distribution based on Records. Communications of the Korean Statistical Society, 18, 102-110. 
Asgharzadeh, A. and Azizpour, M. (2016). Bayesian Inference for Rayleigh Distribution under Hybrid Censoring. International Journal of System Assurance Engineering and Management, 7, 239-249.

Asgharzadeh, A., Abdi, M. and Valiollahi, R. (2013). Analysis of Record Data from the Scaled Logistic Distribution, Journal of Statistical Research of Iran, 10, 41-62.

Asgharzadeh, A., Abdi, M. and Wu, S.J. (2015). Interval Estimation for the Two-parameter Bathtub-Shaped Lifetime Distribution based on Records, Hacettepe Journal of Mathematics and Statistics, 44, 399-416.

Asgharzadeh, A., Abdi, M. and Nadarajah, S. (2016). Interval Estimation for Gumbel Distribution Using Climate Records, Bulletin of the Malaysian Mathematical Sciences Society, 39, 257-270.

Battacharya, P. and Bhattacharjee, R. (2010). A Study on Weibull Distribution for Estimating the Parameters. Journal of Applied Quantitative Methods, 5, 234-241.

Chandler, K.N. (1952). The Distribution and Frequency of Record Values. Journal of Royal Statist Soc., B14, 220-228.

Dey, S., Dey, T. and Kundu, D. (2014). Two-parameter Rayleigh Distribution: Different Methods of Estimation. American Journal of Mathematical and Management Sciences, 33, $55-74$.

Johnson, N.L., Kotz, S. and Balakrishnan, N. (1994). Continuous Univariate Distributions, John Wiley \& Sons, New York.

Khan, H.M.R., Provost, S.B. and Singh, A. (2010). Predictive Inference from a Two-parameter Rayleigh Life Model Given a Doubly Censored Sample, Communications in Statistics - Theory and Methods, 39, 1237-1246.

Kinanci, I., Wu, S.J. and Kus, C. (2017). Confidence Intervals and Regions for the Generalized Inverted Exponential Distribution based on Progressively Censored and Upper Record Data, REVSTAT (to appear).

Mirfarah, E. and Ahmadi, J. (2014). Pitman-Closeness of Preliminary Test and Some Classical Estimators based on Records from Two-parameter Exponential Distribution, Journal of Statistical Research of Iran, 11, 73-96.

Nevzorov, V.B. (1988). Records, Theory of Probability and its Applications, 32, 201-228.

Raqab, M.Z. and Madi, MT. (2002). Bayesian Prediction of the Total Time on Test Using Doubly Censored Rayleigh Data. Journal of Statistical Computation and Simulation, 72, 781-789.

Rayleigh, J.W.S. (1880). On the Resultant of a Large Number of Vibrations of the Some Pitch and of Arbitrary Phase, Philosophical Magazine, 5-th Series, 10, 73-78. 
Soliman, A.A. and Al-Aboud F.M. (2008). Baysian Inference Using Record Values from Rayleigh Model with Application. European Journal of Operational Research, 185, 252-272.

Wu, S., Chen, D. and Chen, S. (2006). Baysian Inference for Rayleigh Distribution under Progressive Censored Sample. Applied Stochastic Models in Business and Industry, 26, 126279.

Zakerzadeh, H., Jafari, A.A. and Karimi, M. (2016). Preliminary Test and Shrinkage Estimations of Scale Parameters for Two Exponential Distributions based on Record Values, Journal of Statistical Research of Iran, 13, 43-58.

\section{Mousa Abdi}

Department of Statistics,

Higher Education Complex of Bam,

Bam, Iran.

email:me.abdi@bam.ac.ir

\section{Akbar Asgharzadeh}

Department of Statistics,

University of Mazandaran,

Babolsar, Iran.

email: a.asgharzadeh@umz.ac.ir 